\title{
Multi-Mission Space Exploration Vehicle (MMSEV) Nosecone Design Optimization
}

XXIV International Conference of the Iberoamerican Society of Digital Graphics Medellin | Colombia

\author{
Maharshi Bhattacharya \\ Harvard Graduate School of Design | United States of America | morbaz@outlook.com \\ Francisco Jung \\ Harvard Graduate School of Design | United States of America | fj2222@columbia.edu
}

\begin{abstract}
This paper addresses ergonomic drawbacks in NASA's modular Multi-Mission Space Exploration Vehicle's (MMSEV) latest prototype, 2B's nosecone, to propose new iteration based on considerations such as mass minimization, visibility maximization, and structural integrity. With $2 \mathrm{~B}$ as a benchmark, and using computational tools typically used in the AEC industry to carry out FEA analysis, comparisons are made with potential design changes. The numerical and visual data such as weight, and stress distribution, provided by the benchmark analysis, served as metrics for comparison and redesign. In turn, this design development exercise attempts to bring together the different design approaches to design, held by humanfactors designers and structural engineers.
\end{abstract}

Keywords: Form; Optimization; Finite Element Analysis; Space-Exploration Vehicle; Stress-Analysis.

\section{INTRODUCTION}

To maximize the number of destinations explored and minimize the number of systems developed, NASA designed a flexible platform that could satisfy multiple demands despite the changing political climate. The MultiMission Space Exploration Vehicle (MMSEV) is a modular spacecraft system primarily comprised of a pressurized core cabin that can be configured with a variety of missionspecific aft and nose augments and life support systems, for in-space and surface exploration, as shown in Figure 1 (Abercromby, Gernhardt, \& Jadwick, 2013; Howard, 2014; Howe, et al., 2015).

NASA plans to conduct human exploration missions to a variety of destinations. The MMSEV is a conceptual platform derived from the Apollo missions as well as the unmanned rovers on Mars that, in the near future, may be used to house and transport astronauts (Bobskill, et al., 2015; Drake, 2009; NASA, 1971; NASA 1972; NASA 1973). The pressurized cabin can be used for in-space missions and surface exploration of planetary bodies, including near-Earth asteroids and Mars. It can be configured with a suit-port, windowed nosecone, and a chassis with wheels to serve as a rover with expedited Extravehicular activity (EVA) capabilities for 14 days to 28 days on the lunar or Martian surface. NASA's overarching ambition has been to design the most efficient modular system through tradeoff studies on habitability, manufacturing costs, and the total wet mass.

However, the prototyped $2 \mathrm{~B}$ glass nosecone for the MMSEV pressurized rover had issues with visibility and foot clearance during the 2009 Desert Research and Technology Studies (DRATS), due to its dome-like geometry (Abercromby, Gernhardt, \& Litaker, 2010). A redesign is due, but human factors designers and structural engineers have two different approaches to design. Therefore, a cohesive design process will be demonstrated in this paper.

Primary design considerations investigated in this paper include minimal mass, maximum visibility, and structural integrity. Minimal mass refers to the bias of the rocket equation toward structures with the lowest weight in materials. Maximum visibility is the overall percentage of fenestrations in areas that crewmembers focus most on. Structural integrity is measured by the normal displacement, Von Mises, stiffness factor, and stress lines. Based on this information, both bottom-up and top-down strategies for design considerations are incorporated to design a novel MMSEV rover nosecone. For the bottom-up approach, the nosecone will be examined through several finite element analyses (FEA) to deduce structural design
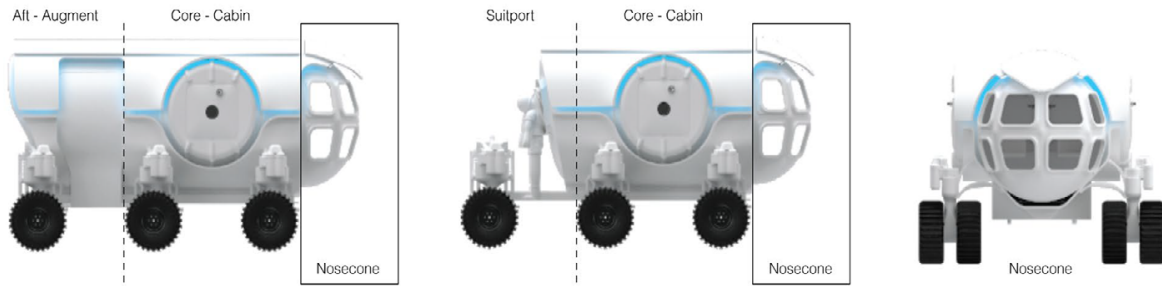

Figure 1: The core cabin can be configured with a variety of mission-specific aft and nose augments and life support systems, for inspace and surface exploration 
elements. These elements include corrugation, thickening, and areas of restriction due to stress concentration. We will attempt to improve on human factors such as visibility with a top-down approach and make design decisions on aperture placement relative to regions of stress concentration deduced from the FEA.

The design requires a structural analysis for the most mass-efficient pressure-retaining cabin, a volumetric study on bare minimum necessities for human habitation for up to 14-days in space based on past HITL evaluations, iterations of different life-support systems configuration, and human factors assessments in microgravity.[5] The unabridged functional requirements and mission-specific objectives will be the design guidelines and success criteria. The specific architecture element to be investigated is the glass nosecone augment that is shared with the rover and hopper platform. The scope of this research is limited to the nosecone section of the pressurized rover. A structural analysis of the existing $2 \mathrm{~B}$ glass nosecone will be done as a benchmark case for potential design iterations to meet or exceed. Design requirements and tradeoffs would be minimal mass, maximum visibility, and structural integrity.

\section{OVERVIEW OF PROTOTYPED AND TESTED SURFACE EXPLORATION VEHICLES}

The structural configuration of the MMSEV has morphed since the 2009 DRATS human-in-the-loop (HITL) field tests to accommodate mission requirements for an asteroid freeflyer under President Obama's asteroid redirect plan. The common cabin is still intended to be the same for all configurations, including the pressurized rover (Litaker, Thompson, \& Howard, 2010). The asteroid free-flyer configuration has many common elements with the rover configuration as they both share the same windowed nosecone and suitport. However, in-space configurations require significant structural attention to the cabin's positive pressure, which entails an affinity for symmetric and curvilinear form. This led to a redesign of the windowed nosecone to meet the structural requirements for an asteroid free-flyer and address the issues of visibility reported in the DRATS 2009 experiment, as shown in Figure 2. The symmetric nosecone with windows was tested in the Neutral Buoyancy Laboratory (NBL) to simulate the microgravity that the crew in an asteroid free flyer would experience.
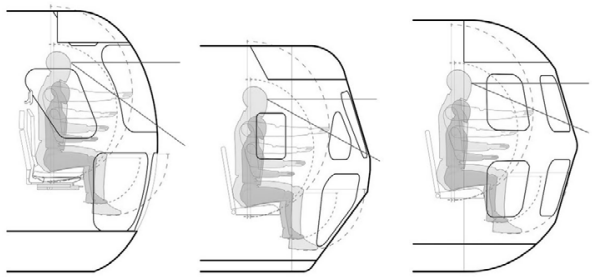

A human factors assessment was done for the symmetric Figure 2: MMSEV Windowed Nosecone (1B, 2A, 2B)

nosecone with windows during the NBL experiment. Visibility was not an issue for the crewmembers in simulated microgravity. However, when the windowed nosecone design was tested in a rover configuration under $1 \mathrm{G}$ conditions, there were issues with foot collision, as shown in Figure 3. Furthermore, the crewmembers had difficulty with peripheral vision in the rover configuration, as evident in Figure 2. The issue with foot collision was mitigated in another redesign of the nosecone, as shown in Figure 4. The resulting form still maintained its symmetric form but had a cylindrical extension to preempt foot collision. Additional redesigns have not been done for window placement as the current MMSEV configuration under development does not require another windowed nosecone.

\section{DESIGN REQUIREMENTS}

Designing the windowed nosecone is a multifaceted process that takes into account tradeoffs between interrelated requirements such as minimal mass, maximum visibility, and structural rigidity. Spacecraft window assemblies generally consist of 4 layers of fused silica for debris, redundancy, pressure, and scratch. Therefore windows should be conservatively placed for mass efficiency. Besides, the windows must be flat paned because curved glass or acrylic, induce visibility issues and added mass due to structural implications for positive pressure on a convex plane. From a top-down perspective, crewmembers require maximum visibility for surface operations. The least amount of replaceable window panels would decrease maintenance and preempt additional weight for spares.

As reported in DRATS 2008 and 2009, the prototyped windowed nosecone prohibited peripheral vision, and the vision of the wheels was unfavorable. As noted from all the Apollo Lunar Roving Vehicle (LRV) missions, lunar regolith's abrasive nature would most likely cause the windows to degrade and require much maintenance. A design consideration would be to digitally create several iterations of the nose cone with different fenestrations that satisfy the aforementioned requirements and run a comprehensive tradeoff study between visibility, mass, and finite element analyses, as shown in Figure 5. It is also noteworthy that SEV 2B was built in Aluminium alloy. However, to bring about drastic mass reduction, we intend

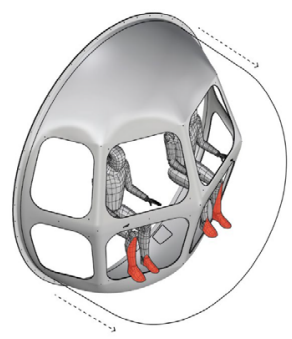

Figure 3: 2B Nosecone Foot Collision

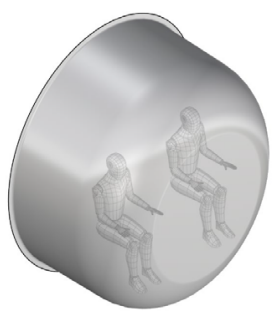

Figure 4: 2B Nosecone Design Update 


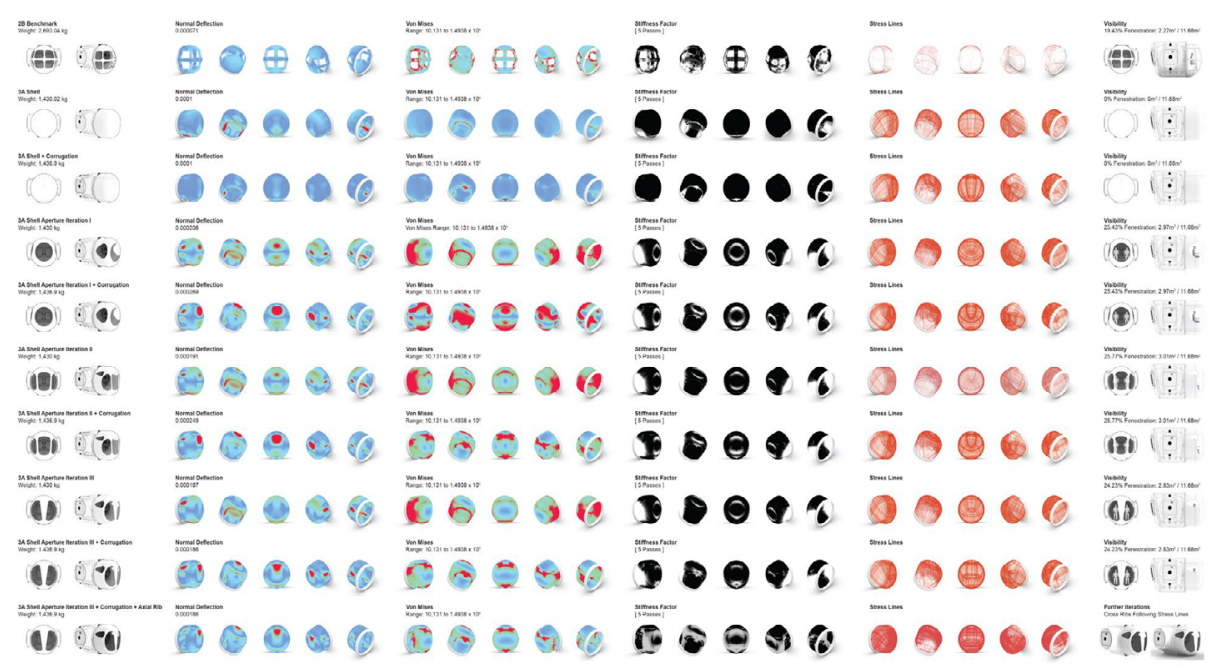

Figure 5: Analysis Matrix

to carry out our analyses with Carbon Fibre as the construction material of choice. It has also been speculated that carbon fibre is poised to gain prominence as a composite ingredient in the space exploration ecosystem (Litaker, Thompson, \& Howard, 2009).

\section{OVERVIEW OF THE EXPERIMENT}

SEV $3 A$ mitigates the foot collision with a more accommodating geometry for a slight structural tradeoff. However, this form factor has never been tested with windows. Therefore, a structural analysis of the existing $2 \mathrm{~B}$ glass nosecone will be done as a benchmark case for potential windowed design iterations with the $3 \mathrm{~A}$ form factor to meet or exceed. Design requirements and tradeoffs would be minimal mass, maximum visibility, and structural integrity. Maximum visibility via unobstructed front-facing windows and generous side windows, are hard requirements but work against the need for minimal mass. Minimum requirements should accommodate the immediate field of vision, at the very least.

\section{EXPERIMENTAL SETUP}

The setup for the experiment consists of three parts, which are:

1. Analysis $\mathrm{I}$, of the benchmark $2 \mathrm{~B}$,

2. Analysis II, of the SEV $3 A$ shell with dead and live load,

3. Analysis III, of the SEV $3 A$ shell with dead and live load and various iterations of window openings.

4. Analysis IV of the iteration with windows with the least maximum deflection was further reinforced with corrugations.

Each of these analyses was carried out on models made on Rhinoceros 3D. Scripts for structural integrity analysis were conceived using two visual programming tools, Grasshopper and Millipede plugins, for Rhinoceros. With minor modifications, the scripts for the analysis of two form factors remained similar, consisting of several parts such as Shell NURB (Non-uniform rational B-spline) to Mesh, Material Definition, Dead and Live Load, Support Definition, Analysis and Optimization, Window Addition and Visual Representation.

The Shell NURB to Mesh component (Figure 6a) converts the model from a NURB surface to a uniformly subdivided mesh, to facilitate sound Finite Element Analysis (FEA). Through the Material Definition component, (Figure $6 \mathrm{~b}$ ) specifications of the shell's material, such as Modulus of Elasticity, Poisson Ratio, Density, Yield Strength, and thickness, are loaded into the script. The Dead and Live Load components (Figure 6c) deal with the dead and live loads acting on the nosecone shell and consider the weight of the ceiling, the floorboard, and two 95th percentile males (98.5 kgs each) with their loads distributed on the floorboard.

The Support definition (Figure 6d) approximates the area on the edge of the nosecone shell that attaches to the core cabin in a SEV. All the data mentioned above is fed into the Analysis and Optimization (Figure 6e) components to carry out the FEA. Within these components, the acceleration due to gravity has been considered $9.81 \mathrm{~m} / \mathrm{s} 2$ with the provision of a regulator to change it as per need. The Window Addition (Figure 6f) component adds the load of the windowpane and frame to various areas of SEV $3 \mathrm{~A}$ where openings are desired in window-arrangement iterations. The Visual Representation (Figure 6g) components are incorporated to visually depict the stress patterns across the nosecone shell through a range of colors, wherein blue represents minimum stress, and red represents maximum stress. Stress lines also show load distribution by way of dense or rare concentration of lines.

Analysis I, of the benchmark, SEV 2B (Figure 7), used al the components mentioned above and the components that calculated the load added by the windowpanes and the frames. Analysis II, of the nosecone shell, SEV 3A, with dead and live load, but without windows, used all the 


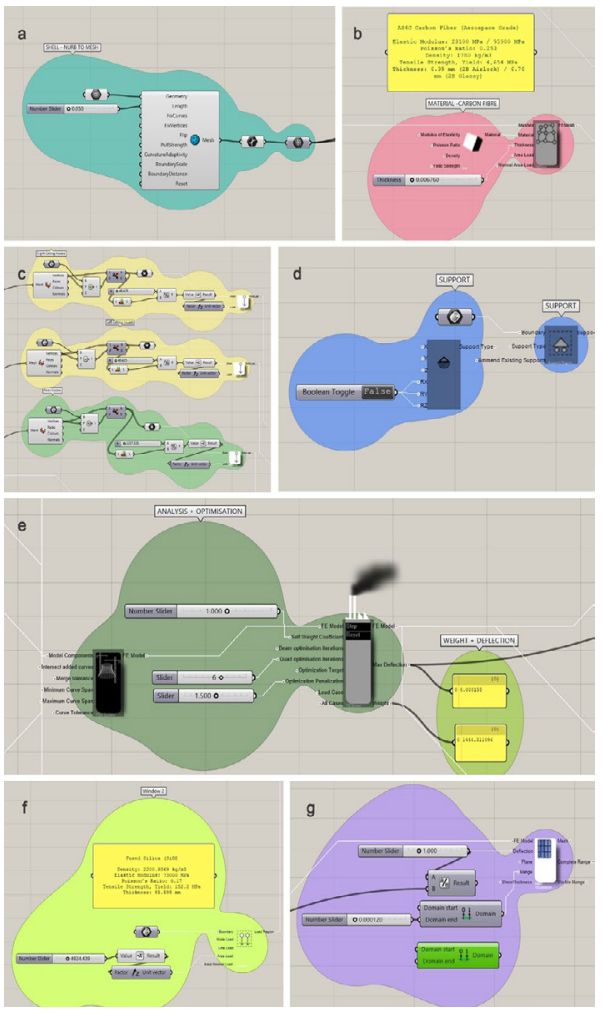

Figure 6a: Shell NURB to Mesh Component

6b: Material Definition

6c: Dead and Live Load Components

6d: Support Definition

6e: Analysis and Optimization Components

6f: Window Addition

6g: Visualization
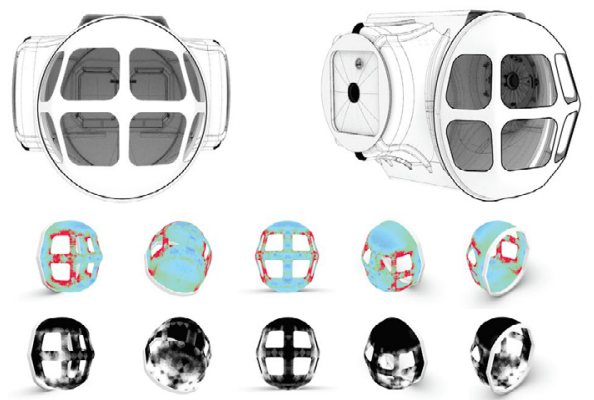

Figure 7: Benchmark, SEV 2B

Analysis I showing Von Mises Stresses and Stiffness Factor

mentioned components, except Window Addition, which was used in analysis III and IV.

\section{PROCEDURES}

Once the script is applied to a nosecone shell, it provides visualizations of structural analytics such as Normal Displacement, Von Mises Stress, Bending Moment,
Principal Stress, and Stiffness Factor. We ran analysis I on SEV $2 B$, which gave us the representations of the stress distribution across the shell, the principal stress lines, and the weight of the shell and maximum deflection. These values helped us set up a range for Normal Displacement and Von Mises Stress, against which we could compare various iterations and the benchmark.

In analysis II, the designated script was run twice: once on the plane, the base shell of SEV 3 A (Figure 8a) and once on a modified SEV $3 A$ with added corrugations (Figure 8b) for structural integrity at the junctions where the ceiling and the floorboard joined the shell. Adding the corrugations was our first design modification to the $3 \mathrm{~A}$ base shell. For this run, we noted down all the analytics as done with the benchmark for comparison.

In analysis III, we furthered the methodology of analysis II and ran the designated script multiple times on the two shells, every time using a different set of windowarrangements, via the Windows Addition component. We again made a note of the different values obtained. To understand the regions of the shell that needed more stiffening, we also ran 4-6 quad optimization iterations, which visually exaggerated the thickness of the said regions to make them stand out.

The stress distribution in the window-arrangement iterations also gave us insight into the regions on the shell where stiffness could be increased. Designing on that, for analysis IV, apart from the windows, we also added more corrugations in regions of plausible stiffness increment as a way to reinforce the structure of the window-arrangement

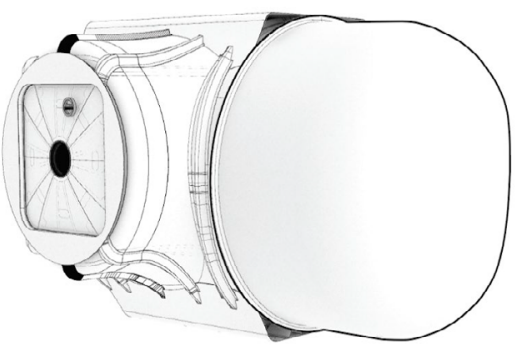

Figure 8a: SEV 3A without Corrugations

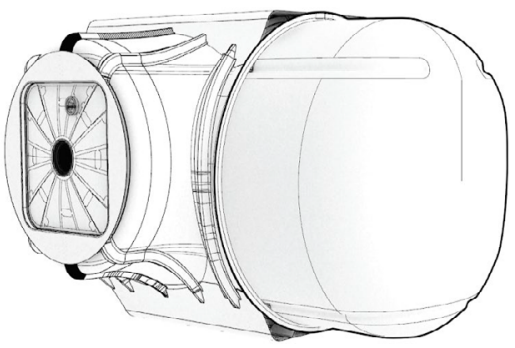

Figure 8b: SEV 3A with Corrugations 

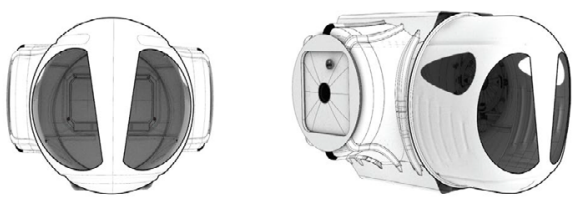

Figure 9: SEV 3A Window Iteration with added Corrugations for Analysis IV

iteration from analysis III, that had the least maximum deflection. (Figure 9)

\section{RESULTS}

In the analysis I, the maximum deflection was $0.071 \mathrm{~mm}$. Taking from this, for any further analysis, we decided to assign a range of 0 to $0.1 \mathrm{~mm}$ to the Normal Displacement, wherein in the visualization, the upper limit of the range is shown in red, values closer to it, in shades of green and values closer to the lower limit, in blue. If a range is not applied, then for each analysis, the maximum displacement for that shell iteration would be shown in red in the visualization, making the visualizations of various iterations incomparable. Similarly, a range was also assigned for Von Mises Stress. The benchmark analysis provided us with a weight as well, which served as a metric for comparison. for all options is provided in Table 1, along with other metrics. Visibility here was calculated as a percentage of the surface area of the nosecone covered by windows.

\section{CONCLUSION}

It is imperative to understand that the aim of this exercise, more than measuring performance by numbers, was to gather information and develop an intuitive understanding of how the stresses act on the nosecone shells with various form factors, based on visual cues provided by the analysis. The objective pursued through this experiment is twofold. Firstly, the iteration used in analysis IV is in no way a final proposal for a structurally robust nosecone shell. It is, however, an example of how the workflow introduced in this experiment can be incorporated to make progress towards a more resolved design.

Secondly, historically, there has been a consistent divide between design and engineering cultures. From a designer's perspective, this experiment has also been an exercise in unpacking the nuances of engineering that play an important role in guiding design decisions; an exercise in reaching out to meet halfway so as to be able to start a dialogue that is equally grounded in aesthetic value as in engineering pragmatism.

Table 1: Comparison of Analyses using mass

\begin{tabular}{|c|c|c|c|c|c|c|c|c|c|}
\hline & MMSEV & $\begin{array}{l}\text { Deflection } \\
\quad(\mathrm{mm})\end{array}$ & $\begin{array}{l}\text { Thickness } \\
(\mathrm{mm})\end{array}$ & $\begin{array}{l}\text { Dead Load (N) } \\
\text { Ceiling + Floor }\end{array}$ & $\begin{array}{c}\text { Live Load(N) } \\
\text { 2 Passengers } \\
\text { 95th Percentile males }\end{array}$ & $\begin{array}{c}\text { Weight }(\mathbf{k g}) \text { of } \\
\text { Nosecone }\end{array}$ & $\begin{array}{c}\text { Weight (kg) } \\
\text { with Windows }\end{array}$ & $\begin{array}{c}\text { Normal } \\
\text { Displacement } \\
\text { Range }\end{array}$ & Visibility \% \\
\hline Analysis I & Benchmark 2A & 0.071 & 13.31 & 316.2 & 1932.57 & NA & 2693.04 & 0 to 0.0001 & $19.43 \%$ \\
\hline \multirow{2}{*}{ Analysis II } & $3 \mathrm{~A}$ & 0.1 & \multirow{2}{*}{6.76} & \multirow{2}{*}{$\begin{array}{c}371.6 \\
(37.9 \mathrm{~kg})\end{array}$} & \multirow{2}{*}{$\begin{array}{l}1932.57 \\
(197 \mathrm{~kg})\end{array}$} & 1430.02 & $\mathrm{NA}$ & 0 to 0.0001 & NA \\
\hline & 3 A Floor + Ceiling Corrugation & 0.112 & & & & 1436.9 & NA & 0 to 0.0001 & NA \\
\hline \multirow{2}{*}{$\begin{array}{c}\text { Analysis III } \\
\text { Window Option } 1\end{array}$} & $3 \mathrm{~A}$ & 0.206 & \multirow{2}{*}{6.76} & \multirow{2}{*}{371.6} & \multirow{2}{*}{1932.57} & 1973.12 & 1973.12 & 0 to 0.00012 & \multirow{2}{*}{$25.43 \%$} \\
\hline & 3A Floor + Ceiling Corrugation & 0.269 & & & & 1980 & 1980 & 0 to 0.00012 & \\
\hline \multirow{2}{*}{$\begin{array}{c}\text { Analysis III } \\
\text { Window Option } 2\end{array}$} & $3 \mathrm{~A}$ & 0.191 & \multirow{2}{*}{6.76} & \multirow{2}{*}{371.6} & \multirow{2}{*}{1932.57} & 1932 & 1932 & 0 to 0.00012 & \multirow{2}{*}{$25.77 \%$} \\
\hline & 3 A Floor + Ceiling Corrugation & 0249 & & & & 1939 & 1939 & 0 to 0.00012 & \\
\hline \multirow{2}{*}{$\begin{array}{c}\text { Analyeis III } \\
\text { Window Option } 3 \\
\end{array}$} & $3 A$ & 0.187 & \multirow{2}{*}{6.76} & \multirow{2}{*}{371.6} & \multirow{2}{*}{1932.57} & 1942 & 1942 & 0 to 0.00012 & \multirow{3}{*}{$24.23 \%$} \\
\hline & 3A Floor + Ceiling Corrugation & 0.186 & & & & 1949 & 1949 & 0 to 0.00012 & \\
\hline \multirow{3}{*}{$\begin{array}{c}\text { Analysis IV } \\
\text { Window Option } 3\end{array}$} & 3A Side Corrugated & 0.155 & 6.76 & 371.6 & 1932.57 & 1444.31 & 1956 & 0 to 0.00012 & \\
\hline & & & & & & & & & \\
\hline & & & & & Von Mises Max Range & 10131 to 1. & $1.4938 \mathrm{e}+6$ & & \\
\hline & & & & & & & & & \\
\hline
\end{tabular}

Something to note here is that for the benchmark (2B), the thickness of the aluminum alloy wall is almost double that of the $3 \mathrm{~A}$. The difference in materials, with differing properties and thickness, has a considerable effect on how the stresses act on the shells. The limitations of aluminum due to its density can be overcome with the strength and lightness of carbon fiber. With the two iterations of analysis II, we get two distinct visualizations of how load-transfer differs across the shells by the addition of a fold or a corrugation. For analysis III, based on this distribution of stresses, through the two iterations, we designed arrangements of windows to maximize visibility while adhering to structural characteristics under applied load, as made evident by the visualizations in analysis II. After selecting the window iteration from analysis III with maximum visibility and the least maximum deflection compared to all $3 \mathrm{~A}$ iterations, we added further corrugation to the shell to increase stiffness. Visually, the amount of red regions were significantly reduced, to have more blue and green regions in this iteration. A comparison of the visibility

\section{ACKNOWLEDGMENTS}

We would like to sincerely thank our faculty and advisor, Professor Sawako Kaijima for the tireless effort she put into our project and the insightful and enlightening experience her teaching has been.

\section{REFERENCES}

Abercromby, A., Gernhardt, M., \& Jadwick, J. (2013). Evaluation of dual multi-mission space exploration vehicle operations during simulated planetary surface exploration. Acta Astronautica, 203-214. doi:10.1016/j.actaastro.2012.02.022

Abercromby, A., Gernhardt, M., Litaker, H. (2010). Desert Research and Technology Studies (DRATS) 2008, Evaluation of Small Pressurized Rover and Unpressurized Rover Prototype Vehicles in a Lunar Analog Environment

Bobskill, M., R., Lupisella, M., L., Mueller, R., P., Sibille, L., Vangen, S., Williams-Byrd, J. (2015). Preparing for Mars: 
Evolvable Mars Campaign: Proving Ground approach, IEEE Aerospace Conference, 2015

Drake, B., G. (Editor) (2009). Human Exploration of Mars Design Reference Architecture 5.0 NASA SP-2009-566-ADD, 2009

Howard, R. (2014). An Alternate Configuration of the Multi-Mission Space Exploration Vehicle. NASA. Retrieved from https://ntrs.nasa.gov/archive/nasa/casi.ntrs.nasa.gov/201400 04223.pdf

Howe, A., Simon. M., Smitherman, D., Howard, R., Toups, L., Hoffman, S. (2015). NASA Evolvable Mars Campaign: Mars Surface Habitability Options. IEEE Aerospace Conference, Big Sky, Montana, USA, 7-14 Mar 2015

Litaker, H., Thompson, S., \& Howard, R. (2009). A Comparison of the Unpressurized Rover and Small Pressurized Rover during a Desert Field Evaluation. Proceedings of the Human Factors and Ergonomics Society Annual Meeting

Litker, H., Thompson, S., \& Howard, R. (2010). Human Habitation in a Lunar Electric Rover during a 14-Day Field Trial. Proceedings of the Human Factors and Ergonomics Society Annual Meeting

Litaker, H., Thompson, S., Szabo, R., Twyford, E., Conlee, C., \& Howard, R. Dual rover human habitation field study. Acta Astronautica, 90(2), 378-390. ISSN 0094-5765

NASA. (1971). Apollo 15 Mission Report. Manned Spacecraft Center, 8-2, 9-8, 14-6. MSC-05161

NASA. (1972). Apollo 16 Mission Report. Manned Spacecraft Center, 8-1, 9-19, 14-115. MSC 07230.

NASA. (1973). Apollo 17 Mission Report. Lyndon B. Johnson Space Center, 9-1, 10-12, 15-25. JSC 07904 